\title{
Ionization of Na Rydberg Atoms by Subpicosecond Quarter-Cycle Circularly Polarized Pulses
}

\author{
T. J. Bensky, G. Haeffler, and R. R. Jones \\ Department of Physics, University of Virginia, Charlottesville, Virginia 22901
}

\begin{abstract}
We report the first observation of ionization of Rydberg atoms by subpicosecond, circularly polarized $\mathrm{THz}$ radiation. The field amplitude in these pulses is non-negligible for only one-quarter of an optical cycle. The experiment is performed in the short-pulse regime, where the duration of the ionizing pulse is shorter than the classical Kepler period of the Rydberg electron. We find that the ionization probability for these atoms is remarkably insensitive to the time-varying polarization of the $\mathrm{THz}$ field.
\end{abstract}

Over the years, multiphoton ionization of atoms has been recognized as a process of fundamental importance for understanding the dynamic interaction between atoms and intense radiation fields. Very recently, a number of investigations have led to the discovery of novel ionization dynamics during the interaction of highly excited Rydberg atoms with strong, subpicosecond pulses of $\mathrm{THz}$ radiation [1-7]. The unipolar electric field in these pulses resembles one-half of an optical cycle of an oscillating electric field so they are commonly referred to as "half-cycle" pulses (HCPs). To date, experimental and theoretical HCP studies have considered only linearly polarized fields. In this configuration, HCPs provide an impulsive unidirectional "kick" that can literally push a Rydberg electron off an atom $[1-3,8]$. Although the ionization dynamics are now relatively well understood for unidirectional fields, previous work with multi-cycle laser [9] and microwave pulses [10], as well as classical intuition, suggests that ionization by subpicosecond far-infrared pulses might proceed very differently with circularly polarized radiation.

In this Letter, we describe the results of the first experiment on the ionization of atoms by subpicosecond circularly polarized $\mathrm{THz}$ pulses. The duration of these pulses is so brief that the electric field vector rotates by only $90^{\circ}$ during the pulse. In the language of classical physics, an electron exposed to this "quarter-cycle" circularly polarized pulse is subjected to a rapidly rotating force in contrast to a unidirectional kick. Therefore, one should expect to observe interesting new ionization dynamics using these novel pulses.

The interaction of atoms with these pulses is also interesting from the point of view of collision physics. A quarter-cycle field pulse bears a strong resemblance to the time-dependent field seen by an atom undergoing a collision with a charged particle, as shown explicitly in Fig. 1. In each case, the field can be described as a combination of a half-cycle cosine pulse along one axis (the transverse collision field) and a "single-cycle" sine component along an orthogonal axis (the longitudinal collision field). In contrast to true collision experiments, the relative velocity, impact parameter, and orientation of the colliding par- ticles can be precisely controlled in a "mock collision" by adjusting the duration, field amplitude, and polarization of the quarter-cycle field pulse.

It is well known that circularly polarized radiation can be decomposed into two waves with orthogonal linear polarizations and a relative delay (or phase shift) of onequarter of an optical period. Similarly, by combining two orthogonal, linearly polarized HCPs with a relative delay of approximately one-half of the HCP duration, the quarter-cycle analog of a circularly polarized pulse
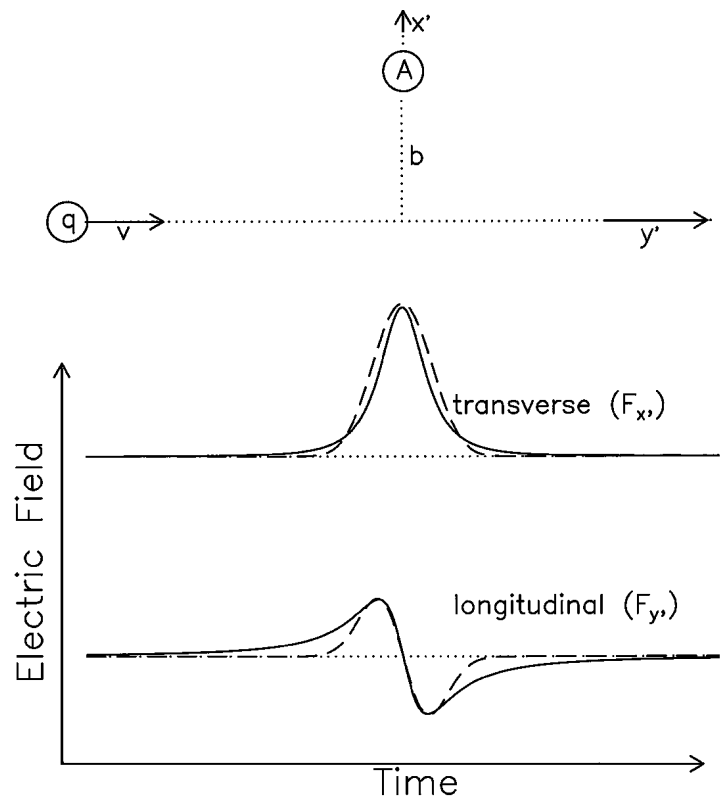

FIG. 1. Top: Schematic of a collision between an atom $A$ and a charged particle $q$ traveling with a relative velocity $v$ at impact parameter $b$. Bottom: The field experienced by the atom during the collision compared to that of a quartercycle circularly polarized pulse. The solid line shows the true collision field while the dashed line is the field produced by two orthogonally polarized HCPs with a relative delay of one-half of their duration [see inset of Fig. 4(A)]. This decomposition of the quarter-cycle circularly polarized field is obtained if the transverse, $\hat{x}^{\prime}$, and longitudinal, $\hat{y}^{\prime}$, collision field directions form a coordinate system which is rotated by $45^{\circ}$ with respect to the $\hat{x}$ and $\hat{y}$ axes of Fig. 2. 
is produced. Furthermore, by selecting different delays between the two, component HCPs, a resultant field pulse with any polarization from linear to circular can be generated.

In the experiment, ground state $\mathrm{Na}$ atoms in a thermal beam are excited through the $3 p_{1 / 2}$ level to a Rydberg state with principal quantum number $15 \leq n \leq 35$ and angular momentum $l=2$, using two tunable nanosecond dye lasers. Approximately $50 \mathrm{nsec}$ after they are produced, the Rydberg atoms are irradiated by a pair of 0.5 psec HCPs. The two HCPs are generated by illuminating two biased GaAs photoconductive switches with two 150 fsec, $790 \mathrm{~nm}$ pulses from an amplified Ti:sapphire oscillator $[1,11]$. The bias fields on the two GaAs wafers are oriented at right angles with respect to each other, producing HCPs with orthogonal linear polarizations [11]. The two HCPs counterpropagate through the Rydberg atom beam between two parallel capacitor plates, as shown in Fig. 2. Approximately $50 \mathrm{nsec}$ after the atoms are exposed to the HCPs, a $50 \mathrm{~V}, 2 \mu \mathrm{sec}$ voltage pulse is applied to the bottom capacitor plate, pushing any ions created by the resultant $\mathrm{THz}$ field through a $50 \times 1000 \mu \mathrm{m}$ extraction slit in the upper capacitor plate toward a microchannel plate detector. The axis of the narrow slit is oriented perpendicular to the HCP propagation axis, giving a temporal resolution of $<200 \mathrm{fsec}$ in this counterpropagating $\mathrm{HCP}$ geometry $[4,7]$.

The number of ions produced by the HCPs is monitored as a function of the peak field in each component HCP as well as the polarization of the resultant field. The peak field is varied by changing the bias voltage on both GaAs switches simultaneously. The polarization of the resultant field is varied continuously by increasing the relative delay between the two $790 \mathrm{~nm}$ laser pulses used to illuminate the GaAs wafers.

The dye lasers, HCPs, and atomic beam are oriented as shown in Fig. 2 to maximize the symmetry of the problem. The two dye lasers propagate parallel to each other and antiparallel to the atomic beam. The two HCPs counterpropagate at right angles to the dye lasers, so that all beams lie in a horizontal plane. The common direction of polarization of the two dye lasers lies along the propagation

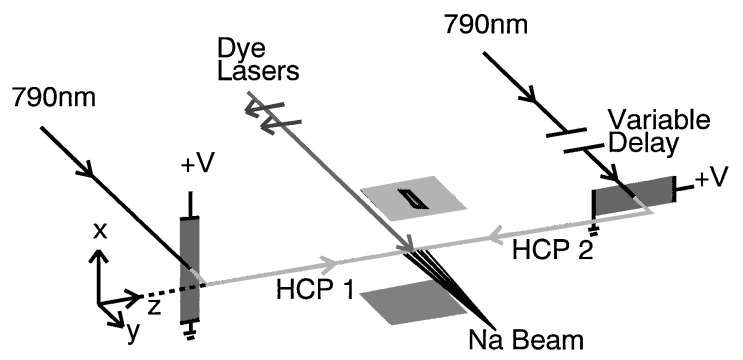

FIG. 2. Schematic of the apparatus showing the relative orientations, propagation directions, and polarizations of the HCPs, lasers, and atomic beams. axis of the two HCPs, $\hat{z}$, and defines the quantization axis for the Rydberg atoms. The polarization vectors of the HCPs are mutually orthogonal and lie in the vertical $x$ $y$ plane. From symmetry, the ionization yield produced by a linearly polarized HCP must be independent of the direction of polarization in the $x-y$ plane.

Measurement of ionization by a quarter-cycle circularly polarized field requires that we detect only those atoms which are exposed to a well-defined relative delay between the two identical, orthogonally polarized component pulses. Periodically during data collection, ionization probability vs field curves are taken for each HCP individually, and the two traces are compared to ensure that the field amplitude in the two pulses agree to better than $10 \%$. In addition, at zero relative delay between the component HCPs, the resultant field seen by the atoms should be a single 0.5 psec HCP that is linearly polarized in the $x-y$ plane at $45^{\circ}$ with respect to the component fields. The amplitude of the resultant field should be $\sqrt{2}$ times larger than the peak field in the component pulses, $F_{0}$. The ionization curves obtained with each HCP alone are compared to those produced with two HCPs at zero relative delay just prior to each circular polarization measurement. The resultant linear field produces the same level of ionization as the individual HCPs at only a fraction of the bias voltage. Typically, this fraction is equal to $1 / \sqrt{2}$ to better than $10 \%$, indicating that the resultant field is, in fact, linearly polarized with an amplitude of, $\sqrt{2} F_{0}$.

Of course, the primary purpose of this experiment is to compare and contrast sub-single-cycle pulse ionization for circular vs linear polarization. In multi-cycle pulse experiments, ionization yields for different polarizations can be compared directly as a function of pulse intensity or peak field. This type of comparison is problematic in the present experiment since the duration and peak field of the combined pulse changes, along with its polarization, as the delay between the component pulses is varied. Instead, ionization yield is monitored as a function of the peak field, $F_{0}$, in the two component pulses. This method does not require that we make any assumptions about the exact temporal shape of the pulse and also lends itself to an intuitive description of the complicated dynamics of the ionization process.

The upper trace in Fig. 3 shows the probability for ionizing the $23 d$ level as a function of $F_{0}$ for zero relative delay, i.e., for a linearly polarized resultant field. The middle and lower traces in Fig. 3 show the ionization yield from the same initial state with "circular" polarization at relative delays of 0.25 and $0.50 \mathrm{psec}$, respectively. The three traces are offset from each other and are identical within the noise level of the experiment. Note that the definition of circular polarization is somewhat ambiguous, and the time-dependent field in both the 0.25 and $0.50 \mathrm{psec}$ cases has a strong resemblance to a quarter-cycle circularly polarized pulse (see insets in Fig. 4). The remarkable feature of the data shown in Fig. 3 is that the ionization 


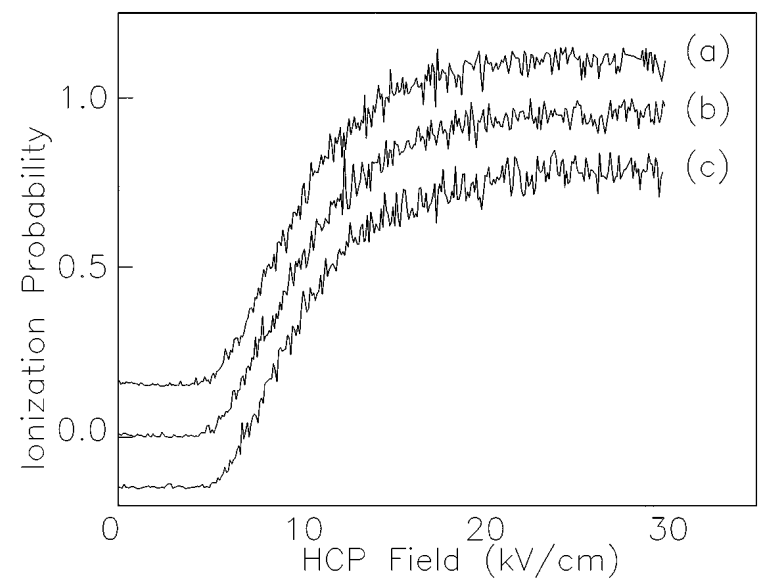

FIG. 3. Ionization probability of the $\mathrm{Na} 23 d$ state as a function of the field amplitude in each of two orthogonally polarized HCPs with relative delays of $(a) 0.0$ psec, $(b)$ $0.25 \mathrm{psec}$, and (c) $0.50 \mathrm{psec}$. The calibration of the field amplitudes is obtained by comparing the ionization yield observed with a single HCP to that calculated classically as suggested in Ref. [14]. The traces are offset from each other for clarity.

level depends only on the peak field in the component pulses, not on the polarization of the resultant field.

Data similar to that shown in Fig. 3 were taken for initial states ranging from $15 d$ to $35 d$. In each case, the similarity between the data taken at the three different relative delays is striking. A least squares algorithm is implemented to

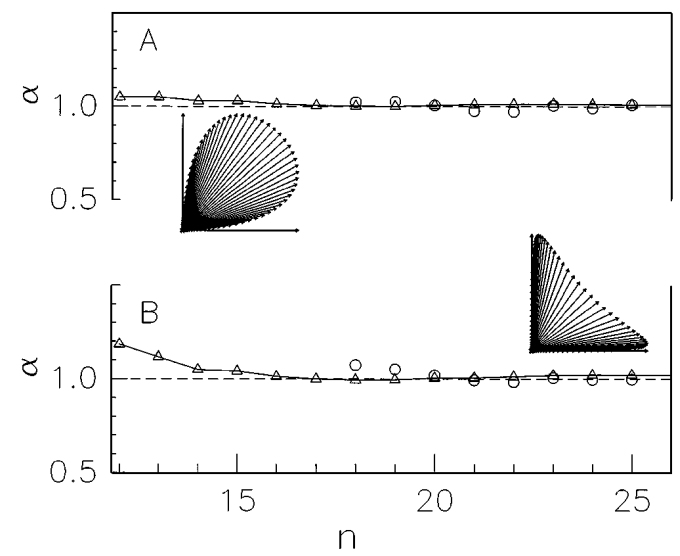

FIG. 4. Ratio $\alpha$ of the component fields, $F_{0}$, which produce the same level of ionization for circular and linear polarization as a function of the principal quantum number of the initial state. The measurements (o) are the average of two independent data runs. The results of the classical simulation are shown as $(\triangle)$ connected with a solid line. The dashed line at $\alpha=1$ is included to guide the eye. (A) shows the results for a relative delay of $0.25 \mathrm{psec}$, while (B) shows the results for $0.50 \mathrm{psec}$. The error bars for the experimental points are typically less than the size of the symbols. Note the clear transition from the short-pulse to the long-pulse regime characterized by the deviation of $\alpha$ from unity near $n=18$. The insets show the resultant field vector as a function of time (clockwise rotation) for the two different cases. make a quantitative comparison of the ionization yields with different polarizations. The fit assumes that, for each initial state, the circular polarization ionization curves differ from the linear ones by a scaling factor of the peak component field, $F_{0}[12]$. Figure 4 shows the average results of the fitting procedure for $n=18$ through $n=25$ for two independent data runs. The dependent axis for the plots is the ratio, $\alpha=F_{0 \text { circ }} / F_{0 \text { lin }}$, which minimizes the value of $\chi^{2}$ between the linear and circular polarization curves. Note that, for all states with $n \geq 20$, the results $\alpha=1.0$ indicate that the linear and circular polarization curves are identical. However, in the 0.50 psec delay data, there is a clear deviation from $\alpha=1.0$ for $n<20$. This deviation is even more apparent for initial states with $n \leq 18$ for both the 0.25 and 0.50 psec data [13].

In previous studies of Rydberg atom/HCP interactions, classical simulations have provided valuable insight into the electron dynamics as well as quantitative agreement with experimental results $[1,2,5,14]$. We find that the current experiment is equally well suited to classical analysis. Specifically, an adaptive step size, fifth-order RungeKutta algorithm has been used to numerically integrate the classical equations of motion for an ensemble of electrons under the influence of two orthogonally polarized HCPs [1]. The electron ensemble simulates the probability distribution of the initial quantum state, and each HCP is modeled as a Gaussian with a 0.5 psec full width at half-maximum (FWHM). The fraction of electrons in the ensemble, whose energy is greater than zero after the combined pulse, gives the ionization probability for a particular pulse amplitude and polarization.

The ionization probability as a function of the peak component pulse field, $F_{0}$, has been computed for initial states, $12 d \leq n d \leq 25 d$, at relative delays of $0.0,0.25$, and $0.50 \mathrm{psec}$ to simulate the experimental conditions. As with the experimental data, the ionization curves for both circular polarization cases $(0.25$ and $0.50 \mathrm{psec})$ are compared to the linear curves $(0.0 \mathrm{psec})$. The ratio of the peak component fields, $\alpha=F_{0 \text { circ }} / F_{0 \text { lin }}$, which gives the best agreement between the circular and linear curves is plotted along with the data in Fig. 4. The agreement between theory and experiment is quite good.

The results of the classical simulation show a clear deviation from the value $\alpha=1$ for values of $n<17$. This same effect is observed in the experimental data at $n \approx 19$. It is not a coincidence that this is the range of principal quantum numbers at which the classical Kepler period of the electron, $\tau_{K}=2 \pi n^{3}$, becomes comparable to the duration of the component HCPs, $\tau_{\mathrm{HCP}}$. The data and theory clearly show that there is a significant change in the ionization dynamics as one moves from the short-pulse regime, $\tau_{\mathrm{HCP}} \ll \tau_{K}(n \gg 15)$, to the long-pulse regime, $\tau_{\mathrm{HCP}} \gg \tau_{K}(n \ll 15)$. We attribute the slight discrepancy in the experimental and theoretical values of $n$ at which the transition between these two regimes occurs to small uncertainties in the temporal shape of the HCP [14]. 
In the long-pulse regime, the field amplitude changes negligibly during one radial oscillation of a classical electron. Therefore, in this adiabatic limit, no energy exchange between the electron and the field occurs. Instead, the field produces a saddle point in the effective binding potential on one side of the atom, allowing the electron to escape from the nucleus without increasing its total energy [15]. Hence, ionization is only sensitive to the height of the saddle point which is determined by the peak resultant field amplitude. For the relative delays of interest in this experiment, $0.0,0.25$, and $0.50 \mathrm{psec}$, the peak resultant fields are $\sqrt{2} F_{0}, \approx 1.2 F_{0}$, and $F_{0}$, respectively. So, at very low values of $n$, one would expect the ratios, $\alpha$ in Fig. 4 , to approach values of $\approx 1.2$ and $\approx 1.4$ for delays of 0.25 and 0.50 psec, respectively.

In contrast, ionization proceeds in the short-pulse regime via energy transfer to the electron through momentum "kicks" due to the component HCPs $[1,2,6,7]$. The results of the classical simulation show that the impulse imparted by a single linearly polarized HCP affects the momentum distribution along the field polarization axis only. The momentum distributions along the other axes remain essentially unchanged and static. Since the ensemble is "kicked" by two orthogonally polarized HCPs, it receives an impulse along two orthogonal axes, but its motions along these two axes are completely decoupled. Therefore, the energy gain from each component kick is independent of the other, and the net ionization yield does not depend on the relative delay between the two pulses. In other words, in the short-pulse regime, ionization is independent of the time-dependent polarization of the net field pulse. In fact, in the short-pulse limit, we find experimentally and theoretically that the ionization yield is independent of the relative HCP delay up to at least several picoseconds for any given HCP amplitude.

In summary, we have created novel $\mathrm{THz}$ pulses with time-varying polarizations, resembling fields that are circularly polarized for only one-quarter of an optical cycle, and used them to observe the ionization dynamics of $\mathrm{Na}$ Rydberg atoms for the first time. In the short-pulse regime, the ionization probability is remarkably insensitive to the time-varying polarization of the field. This insensitivity can be best understood by considering the effect of the orthogonal component fields on the electronic momentum distribution. In essence, the momentum imparted to the electron along one axis does not couple into other Cartesian degrees of freedom. Our simulations indicate that quarter-cycle circularly polarized pulses are capable of producing oriented Rydberg states with very high angular momentum. Therefore these pulses may prove to be an efficient tool for creating circular Rydberg states on a picosecond time scale. In addition, quarter-cycle circularly polarized pulses can be used to simulate controlled ion/ atom collisions.

We gratefully acknowledge the support of the AFOSR, and the Packard Foundation.

[1] R. R. Jones, D. You, and P. H. Bucksbaum, Phys. Rev. Lett. 70, 1236 (1993).

[2] C. O. Reinhold, H. Shao, and J. Burgdorfer, J. Phys. B 27, L469 (1994); C. O. Reinhold et al., J. Phys. B 26, L659 (1993).

[3] R. R. Jones et al., Phys. Rev. A 51, R2687 (1995).

[4] N.E. Tielking and R. R. Jones, Phys. Rev. A 52, 1371 (1995).

[5] A. Bugacov et al., Phys. Rev. A 51, 1490 (1995).

[6] C. Raman et al., Phys. Rev. Lett. 76, 2436 (1996).

[7] R. R. Jones, Phys. Rev. Lett. 76, 3927 (1996).

[8] M. T. Frey et al., Phys. Rev. A 53, R2929 (1996); C. O. Reinhold et al., ibid. 54, R33 (1996).

[9] P. Lambropolous, Adv. At. Mol. Phys. 12, 87 (1976), and references therein; T. J. McIlrath et al., Phys. Rev. A 35, 4611 (1987); P. H. Bucksbaum et al., ibid. 41, 4119 (1990); P. B. Corkum, N. H. Burnett, and F. Brunel, Phys. Rev. Lett. 62, 1259 (1989); P. Dietrich et al., Phys. Rev. A 50, R3585 (1994).

[10] P. Fu et al., Phys. Rev. Lett. 64, 511 (1990); J. A. Griffiths and D. Farrelly, Phys. Rev. A 45, R2678 (1992); P. Kappertz and M. Nauenberg, ibid. 47, 4749 (1993); I. Bialynicki-Birula, M. Kalinski, and J.H. Eberly, Phys. Rev. Lett. 73, 1777 (1994); D. Farrelly and T. Uzer, ibid. 74, 1720 (1995); M. R. W. Bellermann et al., ibid. 76, 892 (1996).

[11] B. I. Greene et al., Appl. Phys. Lett. 59, 893 (1991); D. You et al., Opt. Lett. 18, 290 (1993).

[12] Alternatively, $\alpha$ can be obtained by comparing the component fields at specific ionization fractions (e.g., $10 \%, 50 \%, \ldots)$. The values of $\alpha$ obtained in this way are essentially independent of the ionization fraction used in the comparison. Therefore, the use of a linear fit appears to be justified.

[13] The lower $n$ data are not shown due to difficulties in normalizing the ionization yield for tightly bound states that cannot be ionized with $100 \%$ efficiency with the current apparatus.

[14] N. E. Tielking, T. J. Bensky, and R. R. Jones, Phys. Rev. A 51, 3370 (1995).

[15] T.F. Gallagher, Rydberg Atoms (Cambridge University Press, Cambridge, England, 1994), 1st ed. 